\title{
Is Digital Image Plethysmographic (DIP) Acquisition a Valid New Tool for Preoperative Body Composition Assessment? A Validation by Dual-energy X-ray Absorptiometry
}

\author{
Nicola Di Lorenzo, MD, PhD, FACS ${ }^{1,2}$; Michele Servidio, MD³; Laura Di \\ Renzo, PhD ${ }^{3,4}$; Carmine Orlandi, PhD ${ }^{2,3}$, Giorgio Coscarella, MD ${ }^{1,2}$; \\ Achille Gaspari, MD, FACS ${ }^{1}$; Antonino De Lorenzo, MD, PhD ${ }^{2,3,4}$
}

\author{
${ }^{1}$ University of "Tor Vergata”, Department of Surgery, Rome; ${ }^{2}$ Fondazione Salus, Trasacco; ${ }^{3}$ University \\ of "Tor Vergata", Department of Neuroscience, Division of Human Nutrition, Rome; ${ }^{4}$ I. N. Di. M, \\ National Istitute for Mediterranean Diet and Nutrigenomic Corso Italia, Reggio Calabria, Italy
}

Background: The increasing incidence of obesity and the wider acceptance of laparoscopic surgery, have lead to a 10-fold increase in bariatric operations in the last 10 years. Widely used indices of obesity (weight and body mass index - BMI) cannot adequately distinguish between fat mass (FM), represented by the sum of kilograms (kg) of lipid, and fat-free mass (FFM), inclusive of lean ( $\mathrm{kg}$ of proteins), bone ( $\mathrm{kg}$ of minerals), glycogen, and total body water (TBW), which are important parameters for clinical and physiological studies.

Methods. Anthropometric variables were measured in 19 Caucasian Italian individuals according to standard methods. Body weight $(\mathrm{kg})$ and height $(\mathrm{m})$ were measured, and BMI was calculated as $\mathrm{kg} / \mathrm{m}^{2}$. Body composition was evaluated, with a mean BMI of $25.95 \pm 5.04 \mathrm{~kg} / \mathrm{m}^{2}$, by dual X-ray absorptiometry (DXA) and by digital image plethysmographic (DIP) acquisition with a digital camera. The clear-colored body of the subjects was automatically converted into a front and lateral red-shaped figure, and then through algorithms the 2 pictures were transformed into a nominal volume; body weight was then divided by the estimated volume, so that the body density could be obtained. DXA was used as a comparison to assess fat mass and fat-free mass. Radiation exposure was $<0.6 \mathrm{mSv}$.

Results: Significant positive correlation $(R=0.971$, $P<0.001)$ was found between data of body composition obtained by DXA and DIP.

Conclusions: Body volume assessed using DIP or DXA did not differ. According to this validation study, DIP represents a new promising tool for clinical applications.

Reprint requests to: Nicola Di Lorenzo, Via Salsomaggiore 32, 00135, Rome, Italy. Fax: (+39)-06-0620902926;

e-mail: Nicola.di.lorenzo@uniroma2.it
Key words: Body mass index, body composition, fat mass, lean mass, dual energy X-ray absorptiometry, bariatric surgery, morbid obesity

\section{Introduction}

Obesity has been defined as an excessive accumulation of fat to an extent that health may be impaired, and it has been recognized as a global epidemic by the World Health Organization (WHO). ${ }^{1}$

When dealing with bariatric patients, the concept has been that body mass index $(\mathrm{BMI})>40$ require surgery. This appears to be a rough system to indicate a treatment that results in a defined percentage of morbidity and mortality. However, the vast majority of patients recover uneventfully from their operation, and the final result is a dramatic improvements in quality of life and health status. ${ }^{2}$ Nevertheless, a variable percentage of patients, decreasing from less traumatic to more invasive procedures, do not reach the excess weight loss (EWL) desired, or have a failure in weight loss. These events are related, in some cases, to the operation itself, but more frequently a wrong selection of patients plays a determinant role. According to the WHO cut-off points of BMI for overweight and obesity, $33.4 \%$ of females and $39.5 \%$ of males were overweight $\left(\mathrm{BMI}>25 \mathrm{~kg} / \mathrm{m}^{2}\right)$ and $31.4 \%$ of females and $14.6 \%$ of males were obese $\left(\mathrm{BMI}>30 \mathrm{~kg} / \mathrm{m}^{2}\right){ }^{3,4}$ 
BMI $>40 \mathrm{~kg} / \mathrm{m}^{2}$, or $>35 \mathrm{~kg} / \mathrm{m}^{2}$ with associated comorbidities, is the worldwide method to identify patients eligible for bariatric surgery, but, as described by De Lorenzo et al, ${ }^{5}$ a considerable number of individuals could not be classified as obese based on BMI alone. Therefore, BMI as usually used in population studies to correlate overweight and obesity to morbidity and mortality leads to errors and misclassification.

Our group is currently working on a different method to select patients for different procedures, based on the two pillars: 1) body composition and 2) energy expenditure. Diagnosis, therapy and followup of all subtypes of obesity must not be based on the "body weight" parameter alone, but on body composition parameters. Misclassification of obesity, at an individual as well as a population level, has occurred because information, assumptions and predictive formulas of body composition were usually performed only on Caucasians. There is a need for cross ethnic population studies on body composition. ${ }^{6}$ Measurements of direct body fat mass percentage would be a better tool for diagnosing any kind of obesity. An effort should be made to provide such data. A correct diagnosis of fat content and proportion is important to estimate not only overweight and obesity but also risk factors for cardiovascular and metabolic diseases. Therefore, screening for proportion of body fat in individuals with normal or slightly elevated BMI is important in the prevention of diabetes and cardiovascular diseases.

Estimation of overweight and obesity presupposes calculation of body mass component proportion and risk factors for diseases, such as type 2 diabetes, dyslipidemia, impaired glucose tolerance and cardiovascular disease. It has been reported that the risk of coronary artery disease increases with the severity of obesity, especially abdominal, when the waist-to-hip ratio (WHR) is $>0.90$ in males and $>0.85$ in females. ${ }^{7,8}$ However, as shown by Busetto et al, ${ }^{9}$ WHR cannot be considered an index of visceral/subcutaneous fat proportion in obese patients, especially in obese women. Assessment of absolute amount of fat and its proportion are important.

In clinical practice, the measurements of weight and height to perform BMI calculation are simple, but not sufficient to detect changes in proportion of fat and lean mass. To avoid misclassifications, measurements of body fat percentage would be a better tool for diagnosing obesity. Body composition can be measured at different levels, ranging from atomic, molecular, cellular, tissue, to whole body level and each information obtained at one level can be transformed into other levels, using chemical constants or experimentally and statistically derived constants or relationships. ${ }^{10}$ However, all these techniques are rather expensive and sometimes out of reach of the financial capabilities of private practitioners or small institutions.

The purpose of the present study was to define a new and low cost approach based on digital image plethysmographic (DIP) acquisition to assess body volume and body fat mass proportion. Because density is defined as mass divided by volume and weight is easily obtained, the outcomes of body density are cost-effective. We propose a novel method for accurately estimating the relative level of body fat and lean proportion, by non-invasive body composition analysis. Our data suggest that application of DIP in body composition measurement can screen and identify obese subjects, and follow them after bariatric surgery.

\section{Materials and Methods}

\section{Participants}

A total of 19 Caucasian Italian subjects (10 males and 9 females), with age $37 \pm 13$ SD years and with BMI $25.95 \pm 5.04 \mathrm{~kg} / \mathrm{m}^{2}$ were examined. They were randomly selected from 3,000 participants of ongoing studies on body composition and energy metabolism at the Unit of Human Nutrition of the Tor Vergata University (Rome, Italy), and at Fondazione Salus (Italy). Informed consent was obtained from all the participants before the beginning of the study, according to the guidelines of Medical Ethics Committee of the University.

\section{Anthropometric Measurements}

Anthropometric parameters for all participants were measured according to standard methods: body weight, height, and hip and waist circumferences. Individuals were instructed to take off their clothes and shoes before any measurements were taken. Body weight $(\mathrm{kg})$ was measured to the nearest $0.1 \mathrm{~kg}$, using a balance scale (Invernizzi, Rome, Italy). Height (m) 


\section{Di Lorenzo et al}

was measured using a stadiometer to the nearest 0.01 m (Invernizzi, Rome, Italy). BMI was calculated using the formula: weight $(\mathrm{kg}) /$ height $\left(\mathrm{m}^{2}\right)$.

\section{Dual X-ray Absorptiometry (DXA)}

Total body composition was assessed by dual-energy x-ray absorptiometry (DXA) (Lunar DPX). The technique combined a total body scanner, an X-ray source, an internal wheel to calibrate the bone mineral compartment, and an external lucite/aluminium phantom to calibrate the fat compartment. Standard DXA quality control and calibration measures were performed prior to each testing session. The subjects were given complete instructions on how the testing procedure would occur. The subjects were instructed not to exercise within $24 \mathrm{~h}$ of the test. Individuals were required to remove all clothing except undergarments, including shoes, socks and jewels, prior to being positioned on the DXA table. They lay in supine position on DXA, without moving for 15 minutes while the DXA scan recorded their results. The entire body was scanned beginning at the top of the head and moving in a rectilinear pattern down the body to the feet. Radiation exposure was $<0.6 \mathrm{mSv}$.

\section{Digital Image Plethysmographic (DIP) Acquisition}

All participants were instructed to come to at the Unit of Human Nutrition of the Tor Vergata University (Rome), with clear shaded underwear and/or clear bathing outfits. One front and one lateral digital image of each subject were taken with a digital web camera, having the subject standing against a dark non-reflective wall, surrounded by a white frame of 1 inch $(2.5 \mathrm{~cm})$ thickness, of fixed and known dimensions (1.80 meters x 2.30 meters). The clear-colored body of the subject obtained by DIP was automatically digitally-converted into a front and lateral red-shaped figure. Property algorithms were able to transform the two pictures into a nominal volume, which dividing the weight produces body density data, used to determine, by equation, body fat mass (FM) percentage.

A rotating circular platform of $100 \mathrm{~cm}$ diameter was automatically rotated by a low power, low voltage electrical motor, with a rotating speed of one revolution per minute. The platform is mounted on ball- bearings and able to sustain $>300 \mathrm{~kg}$. It is mounted under a square $150 \mathrm{~cm} \times 250 \mathrm{~cm}$ frame, white colored, backed by black-colored lining, to create a high contrast background, as shown in Figure 1.

At the $0^{\circ}$ and $90^{\circ}$ interval, two reed sensors are fitted under the revolving platform to automatically trigger the acquisition of one front and one lateral full body digital image (Figures 2 and 3). The platform is also fitted with two shoes, to block the subject always in the same position for a repetitive and correct data acquisition.

Positive rotation driven by a 12 VDC motor, and synchronized image acquisition are governed via a standard RS 232C protocol by Windows-based software. The software also manipulates the captured images, and computes automatically the relative surface of the projection of the body against the black background of the subject that is standing upright, with abducted legs.

The entire process of rotation and image acquisition takes about 45 seconds. Volume was assessed by transforming the digital picture into body volume.

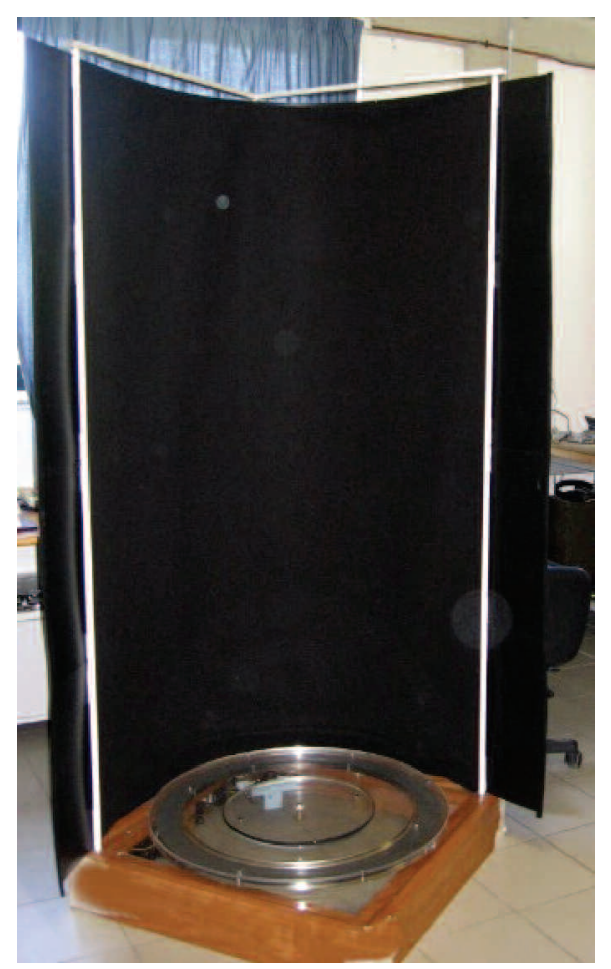

Figure 1. Rotating circular platform is mounted on ballbearings and able to sustain more than $300 \mathrm{~kg}$. It is mounted under a square $150 \times 250 \mathrm{~cm}$ frame, white-colored, backed by black-colored lining, to create a high contrast background. 


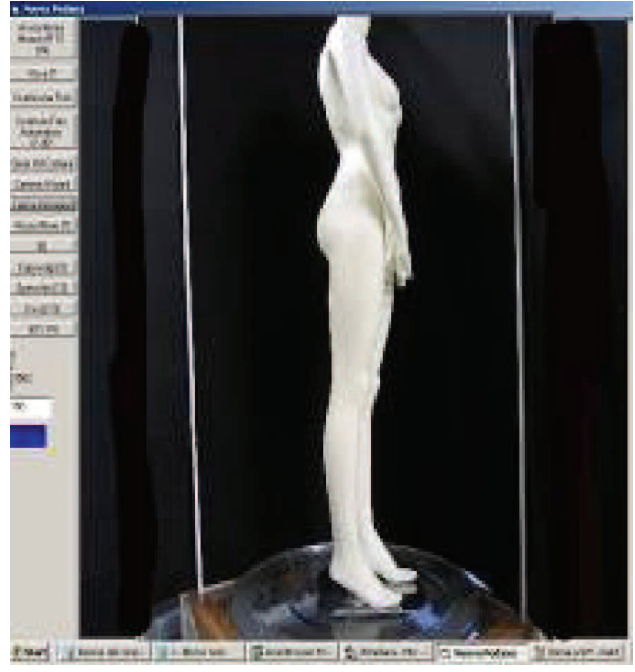

Figure 2.

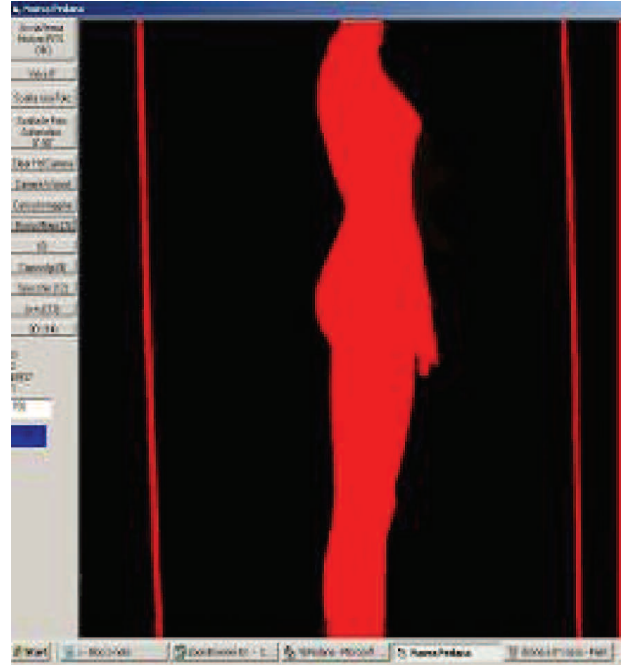

Figure 3.

Figure 2 and Figure 3. The clear-colored body of the subject is automatically digitally-converted into a front and lateral red-shaped figure. The software manipulates the captured images, and computes automatically the relative surface of the projection of the body against the black background of the subject that is standing upright, with abducted legs.

\section{Statistical Analysis}

According to the statistician's evaluation, the number of analyzed subjects was valid to evaluate the hypothesis. Descriptive values are expressed as means and standard deviation (SD). Linear regression and correlation were used to evaluate the relationship among variables. Statistical analysis was performed using Systat 10 statistical package (SPSS Chicago, IL). A value of $P<0.05$ was considered significant.

\section{Results}

Descriptive variables of the 19 Caucasian Italian study participants (10 males and 9 females) - age, weight, height, BMI, and body fast mass (FM) in kg and $\%$, are shown in Table 1 . The means of body fat mass (FM) and fat mass percentage (FM\%) by DXA and body fat mass (FM) and fat mass percentage (FM\%) by DIP were compared.

Age and sex distribution did not differ between the groups. Mean age of participants was $37 \pm 13$ years, and mean BMI was of $25.95 \pm 5.04 \mathrm{~kg} / \mathrm{m}^{2}$. According to the study design, the participants were classified not only on the basis of their BMI but also on the basis of their body composition. Mean fat mass (FM) of individuals measured by DXA was $22.07 \pm 11.05 \mathrm{~kg}$, and body composition measured by DIP showed a fat mass of $24.6 \pm 9.92 \mathrm{~kg}$. There were no significant differences between DXA and DIP in estimating body fat mass (FM) proportion.

A linear regression analysis was performed to compare body fat mass percentage measured by DIP and by DXA. As shown in Figure 4 a significant positive correlation was obtained between fat mass proportion measured by DIP and DXA $(\mathrm{R}=0.971$, $P<0.001)$. Fat mass proportion obtained by DXA was also compared with BMI calculated as $\mathrm{kg} / \mathrm{m}^{2}$. Figure 5 showed no significant correlation between BMI and DXA body fat mass $(\mathrm{R}=0.32, P=0.045)$.

Table 1. Body composition parameters of 19 Caucasian Italian individuals

\begin{tabular}{lcc}
\hline Studied Individuals & MEAN & SD \\
\hline Age (years) & 37 & 13 \\
Weight $(\mathrm{kg})$ & 73.5 & 16.77 \\
Height $(\mathrm{m})$ & 1.68 & 0.08 \\
BMI $\left(\mathrm{kg} / \mathrm{m}^{2}\right)$ & 25.95 & 5.04 \\
FM DXA $(\mathrm{kg})$ & 22.07 & 11.05 \\
FM DXA \% & 28.99 & 11.80 \\
FM DIP $(\mathrm{kg})$ & 21.6 & 9.92 \\
FM DIP \% & 29.4 & 9.92 \\
\hline
\end{tabular}

FM DIP = body fat mass measured by DIP. 


\section{Di Lorenzo et al}

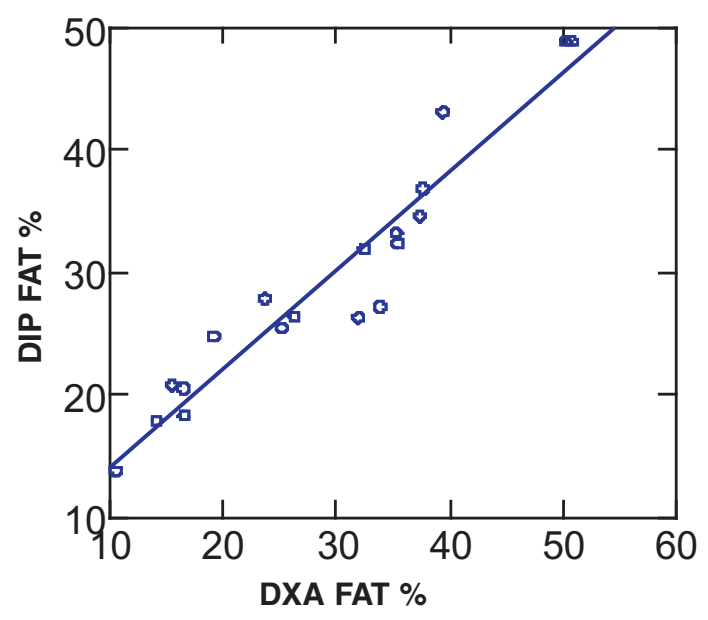

Figure 4. Linear regression by body fat mass percentage measured by DXA and body fat mass estimated by DIP.

\section{Discussion}

Knowledge of body surface area (BSA) of the human has important applications in medical treatment, biomechanics and other engineering processes. Several expressions have correlated BSA with direct measurements of body mass and body height, ${ }^{11-15}$ but each method has some bias. Therefore, it appears necessary to address a new more accurate BSA assessment in overweight and obese individuals. The dual-energy X-ray absorp-

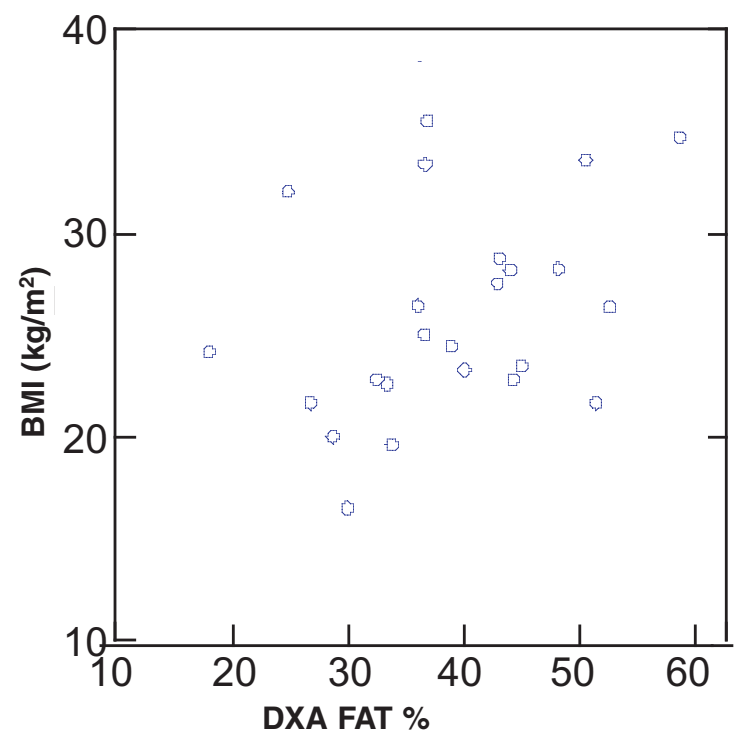

Figure 5. Linear regression by fat mass percentage measured by DXA and calculated by BMI. tiometry (DXA) device ${ }^{16}$ is highly reliable, and there is good agreement $(\sim 0.4 \% \mathrm{FM}$ difference) between \%FM estimates obtained by hydro-densitometry, currently considered a "gold standard" of body composition analysis. ${ }^{17}$ However, these two most accepted methods showing reliability, sensitivity and precision, are not applicable as a daily routine: there are several limitations to the hydrostatic weighing technique because it requires considerable cooperation, given that multiple trials need to be performed. In addition, elderly people, children, physically-challenged persons, and individuals with certain diseases may not be able to comply with standardized procedures.

As an alternative, density can be assessed with DXA. This method is based on a three-compartment model that divides the body into total-body mineral, mineral-free lean, and fat tissue masses. The precision of DXA in measuring \% FM is estimated to be within $1.2 \%$. Suh et $\mathrm{al}^{18}$ showed that lumbar region mass distribution, as measured by DXA, is a better predictor of Visceral Abdominal Tissue (VAT) than the waist circumference and WHR or BMI in obese postmenopausal women. It has also been shown that DXA, possibly combined with anthropometry, offers a good alternative to computed tomography (CT) for the prediction of visceral fat also in the elderly, ${ }^{19}$ and DXA provides an accurate measurement of VAT in addition to the total body composition determination in obese individuals. DXA fat proportion estimates may be useful in the early detection of individuals with abdominal/visceral obesity. ${ }^{20,21}$ Weight management can help reduce the number of people at risk for diabetes and reduce the risk for complications and premature mortality. ${ }^{22}$

Currently, bariatric surgery remains the most effective means to achieve and maintain excess weight loss (EWL) in morbid obesity (BMI $>40$ or BMI $>35$ in the presence of significant co-morbidities). Various bariatric surgery techniques are used. The intragastric balloon may be used for temporary weight reduction for mild or moderate obesity, or for preoperative weight loss in super-obesity. ${ }^{23}$ Gastric banding procedures induce considerable weight loss in obese subjects. ${ }^{24,25}$ Gastric bypass, the most frequent operation performed in USA, is more invasive and still more efficient, with satisfactory $>60 \%$ EWL. ${ }^{2,26}$ Biliopancreatic diversion of Scopinaro et $\mathrm{al}^{27,28}$ is a mixed and complex operation that, in association with nutrient supplementation to 
avoid nutritional deficiency, has shown the most effectiveness in weight loss and permanent normalization of serum glucose, triglyceride, and cholesterol levels, with results maintained for $>10$ years follow-up.

Current selection criteria for bariatric operations do not include patients with $\mathrm{BMI} \leq 35$ or $<40$ without co-morbidities. However, due to the possible misclassification of obesity based on BMI alone, it appears necessary to formulate a diagnosis based on proportion of body fat mass.

This paper presents the first results of a validation study of a new promising digital image acquisition (DIP) technique using a digital web camera to measure body volume and body fat proportion, adopting DXA as the reference standard. ${ }^{29,30}$ We have evaluated a new system based on digital image scanning, that is rapid with a forecasted low cost, totally non-invasive and very promising in the accuracy of prediction of fat mass proportion. Its working principle is simple and applicable in any circumstance because it does not demand special training or cooperation by the examined subject, other than being able to stay still in the upright position with abducted legs for 1 minute.

Body composition of all participants in the study was measured by dual X-ray absorptiometry (DXA) and by digital imagining acquisition (DIP) techniques. By linear regression analysis, a significant positive correlation $(\mathrm{R}=0.971, \quad P<0.001)$ was obtained between body fat mass percentage measured by DIP and body fat mass percentage measured by DXA. On the other hand, no significant correlation was obtained when comparing body fat mass values by DXA or DIP to the BMI values of the entire group, demonstrating the potential misclassification of obesity diagnosis based on BMI alone. According to this validation study, DIP represents a new promising technique, useful as a tool for clinical applications. This technique may be a good tool for suggesting surgical treatment to obese patients, identified according to FM proportion and BMI, and following-up the excess weight loss in the short- or long-term after bariatric operations.

\section{References}

1. Deitel M. Overweight and obesity worldwide now estimated to involve 1.7 billion people (Editorial). Obes Surg 2003; 13: 329-30.
2. Buchwald H, Williams SE. Bariatric surgery worldwide 2003. Obes Surg 2004; 14: 1157-64.

3. WHO Physical Status: The use and interpretation of anthropometry. Technical Report Series 854. WHO, Geneva, Switzerland, 1995.

4. Obesity: Preventing and managing the global epidemic. Report on a WHO Consultation of Obesity WHO/NUT/NCD/98.1, Geneva, Switzerland, June 35, 1997.

5. De Lorenzo A, Deurenberg P, Pietrantuono $\mathrm{M}$ et al. How fat is obese? Acta Diabetol 2003; 40: S254-S257.

6. Deurenberg P, Deurenberg-Yap M. Validity of body composition methods across ethnic population groups. Acta Diabetol 2003; 40: S246-S249.

7. Katzel LI, Sorkin KD, Colman E et al. Risk factors for exercise-induced silent myocardial ischemia in healthy volunteers. Am J Cardiol 1994; 74: 869-74.

8. Lohman TG, Roche AF, Martorell R. Anthropometric Standardization Reference Manual: Human Kinetics. Champaine, IL 1998.

9. Busetto L, Baggio MB, Zurlo F et al. Assessment of abdominal fat proportion in obese patients: anthropometry versus computerized tomography. Int J Obes 1992; 16: 731-6.

10 Wang ZM, Pierson RN, Heymsfield SB. The fivelevel model: a new approach to organize body composition research. Am J Clin Nutr 1992; 56: 19-28.

11. Wang J, Hihara E. A unified formula for calculating body surface area of humans and animals. Eur J Appl Physiol 2004; 421: 1074-9.

12. Sardinha LB, Carnero EA, Martins S et al. Body surface area estimation and its impact on predicting appendicular skeletal muscle mass with a mechanistic model based on the reference Man. Acta Diabetol 2003; 40: S29-S31.

13. Du Bois D, Du Bois EF. A formula to estimate the approximate surface area if height and weight be known. Arch Intern Med 1916; 17: 863-71.

14. Livingston EH, Lee S. Body surface area prediction in normal weight and obese patients. Am J Physiol Endocrinol Metab 2001; 281: 586-91.

15. Fuller NJ, Laskey MA, Elia M. Assessment of the composition of the major body regions by dual-energy X-ray absorptiometry (DEXA) with special reference to limb muscle mass. Clin Physiol 1992; 12: 253-66.

16. Prior BM, Cureton KJ, Modlesky CM et al. In vivo validation of whole body composition estimates from dual-energy X-ray absorptiometry. J Appl Physiol 
Di Lorenzo et al

1997; 83: 623-30.

17. Durin JUGA, Wormersly J. Body fat assessment from total body density and its estimation from skin fold thickness: Measurement of 481 men and women aged 16-72. Br J Nutr 1974; 32: 77-97.

18. Suh YS, Kim DH, Lee IK. Usefulness of lumbar AP spine DXA for measuring the percentage of perilumbar regional fat and predicting visceral fat in obese postmenopausal women. Nutrition 2002; 18: 84-5.

19. Snijder MB, Visser M, Dekker, JM et al. The prediction of visceral fat by dual-energy-X-ray absorptiometry in the elderly: a comparison with computed tomography and anthropometry. Int J Obes 2002; 26: 984-93.

20. Bertin E, Marcus C, Ruiz JC et al. Measurement of visceral adipose tissue by DXA combined with anthropometry in obese humans. Int J Obes 2000; 24 : 263-70.

21. Park YW, Heymsfield SB, Gallagher D. Are dualenergy X-ray absorptiometry regional estimates associated with visceral adipose tissue mass? Int J Obes 2002; 26: 978-83.

22. Prevalence of overweight and obesity among adults with diagnosed diabetes - United States, 1988-1994 and 1999-2002. Centers for Disease Control and Prevention. JAMA 2005; 293: 546-7.

23.Al-Momen A, El-Mogy I. Intragastric balloon for obesity: a retrospective evaluation of tolerance and efficacy. Obes Surg 2005; 15: 101-5.
24. Angrisani L, Favretti F, Furbetta F et al. Italian Group for Lap-Band System ${ }^{\circledR}$ : Results of multicenter study on patients with BMI $\leq 35 \mathrm{~kg} / \mathrm{m}^{2}$. Obes Surg 2004; 14 : 415-8.

25. Watkins BM, Montgomery KF, Ahroni JH et al. Adjustable gastric banding in an ambulatory surgery center. Obes Surg 2005; 15: 1045-9.

26. Rosenthal RJ, Szomstein S, Kennedy CI et al. Laparoscopic surgery for morbid obesity: 1,001 consecutive bariatric operations performed at the bariatric institute, Cleveland Clinic Florida. Obes Surg 2006; 16: 119-24.

27. de Luis DA, Pacheco D, Izaola O, et al. Early clinical and surgical results of biliopancreatic diversion. Obes Surg 2005; 15: 799-802.

28. Scopinaro N, Marinari GM, Camerini GB et al. Specific effects of biliopancreatic diversion on the major components of metabolic syndrome: a long-term follow-up study. Diabetes Care 2005; 28: 2406-11.

29. Brozek J, Grande F, Anderson JT et al. Densitometric analysis of body composition: revision of some quantitative assumptions. Ann NY Acad Sci 1963; 110: 113-140.

30. Siri WE. Body composition from fluid spaces and density: analysis of methods. In: Brozek J, Henschel A, eds. Techniques for Measuring Body Composition. Washington, DC: National Academy of Sciences 1961: 223-44.

(Received January 17, 2006; accepted March 10, 2006) 